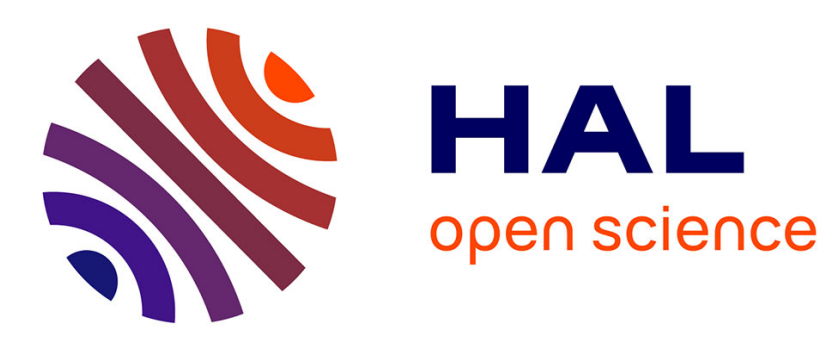

\title{
Experimental study of trapdoor problem in 3 dimensions with X-ray CT - transition from plane strain to $3 \mathrm{D}$ behavior
}

\author{
Bastien Chevalier, Jun Otani, T Mukunoki
}

\section{- To cite this version:}

Bastien Chevalier, Jun Otani, T Mukunoki. Experimental study of trapdoor problem in 3 dimensions with X-ray CT - transition from plane strain to 3D behavior. 14th IACMAG International Conference, Sep 2014, Kyoto, Japan. 10.1201/b17435-56 . hal-01126774

\author{
HAL Id: hal-01126774 \\ https://hal.uca.fr/hal-01126774
}

Submitted on 6 Mar 2015

HAL is a multi-disciplinary open access archive for the deposit and dissemination of scientific research documents, whether they are published or not. The documents may come from teaching and research institutions in France or abroad, or from public or private research centers.
L'archive ouverte pluridisciplinaire HAL, est destinée au dépôt et à la diffusion de documents scientifiques de niveau recherche, publiés ou non, émanant des établissements d'enseignement et de recherche français ou étrangers, des laboratoires publics ou privés. 


\title{
Experimental study of trapdoor problem in 3 dimensions with $\mathrm{X}$-ray $\mathrm{CT}$ - transition from plane strain to $3 \mathrm{D}$ behavior
}

\author{
B. Chevalier \\ Clermont Université, Université Blaise Pascal, Institut Pascal, Clermont-Ferrand, France \\ CNRS, UMR 6602, Institut Pascal, Aubière, France \\ J. Otani \& T. Mukunoki \\ X-Earth Center, Graduate School of Science and Technology, Kumamoto University, Japan
}

\begin{abstract}
This paper present an experimental study of the trapdoor problem in 3-dimensions using X-ray computed tomography. Trapdoor tests were performed on layers of glass beads of different sizes. Different trapdoor shapes varying from square to rectangular shape were also tested. The trapdoor tests were analyzed in terms of load transfer (force) and in terms of propagation of the disturbance created in the layer by the trapdoor movement. The results revealed the existence of a transition between a 2-dimesional behavior and a 3-dimensional behavior in the trapdoor problem, as the aspect ratio of the trapdoor increased.
\end{abstract}

\section{INTRODUCTION}

Granular materials are subject to arching effect. The phenomenon of arching appears in such materials when a localized settlement appears at the bottom of a layer. Practically in geotechnical engineering, such settlements can be the result of many causes: manmade excavation like tunneling, mining, or natural subsidence like sinkholes. The behavior of granular material in relation to arching was initially highlighted by Terzaghi (1936) and since then widely studied (Terzaghi 1936, Tanaka \& Sakai 1993, Vardoulakis et al. 1981, Dewoolkar et al. 2007, Chevalier et al. 2012, Pardo \& Sáez 2014). There are two main aspects of arching in granular material that are of high interest for geotechnical engineering: in which proportion the loads - self weight and overload - located above settlement area will be transferred to stable areas; how far upwards in the granular layer the disturbance due to the settlement will propagate. Both questions are difficult to answer because of the numerous parameters involved in the problem definition and which may influence the response of a given material in a particular arching situation.

Many researchers have studied the arching phenomenon using the trapdoor problem. The trapdoor problem consists in artificially creating a localized settlement at the bottom of a granular layer by moving downward a small part of its supporting plate. In the trapdoor problem, the question of the load transfer can be relatively easily investigated as soon as one can measure the forces applied to the trapdoor during its movement. However, the second question of the disturbance propagation is less trivial unless the case of plane strain condition trapdoor problem is considered.
The difficulty of investigating disturbance propagation is the need to see and measure the kinematics of the granular layer during arching. X-Ray Computed Tomography (CT) is an efficient measurement technique for non destructive investigations of volume of materials in 3-dimensions. Chevalier \& Otani (2011) studied trapdoor problem with both X-Ray CT and Discrete Element Method with model granular material (glass beads) to evaluate the possibilities of both approaches. In this previous study, only one size of glass beads and one size of trapdoor were tested.

In this study, trapdoor problem tests are conducted on glass beads layer of different sizes, and with trapdoors of different size and shapes. Load transfers in the granular material were measured and the propagation of the disturbance within the granular layer was quantified with X-Ray CT.

\section{MATERIAL AND METHODS}

\subsection{Experimental apparatus}

The trapdoor problem tests were conducted in an acryl cylindrical box of diameter, $240 \mathrm{~mm}$ (Fig. 1). In the centre of the bottom plate of the box, a trapdoor made of an aluminium alloy could be placed presenting different aspect ratio depending on the case studied: $40 \times 40 \mathrm{~mm}$ (aspect ratio 1:1), $80 \times 40 \mathrm{~mm}$ (aspect ratio $2: 1$ ) or $120 \times 40 \mathrm{~mm}$ aspect ratio $3: 1$ ), and respectively called $T_{1}, T_{2}$ and $T_{3}$. The trapdoor is supported by a steel support with 4 vertical rods that can slide vertically in the steel support. One (with $\mathrm{T}_{1}$ and $\mathrm{T}_{2}$ ) or two (with $\mathrm{T}_{3}$ ) load cells are placed between trapdoor and steel support to measure the vertical force applied by 


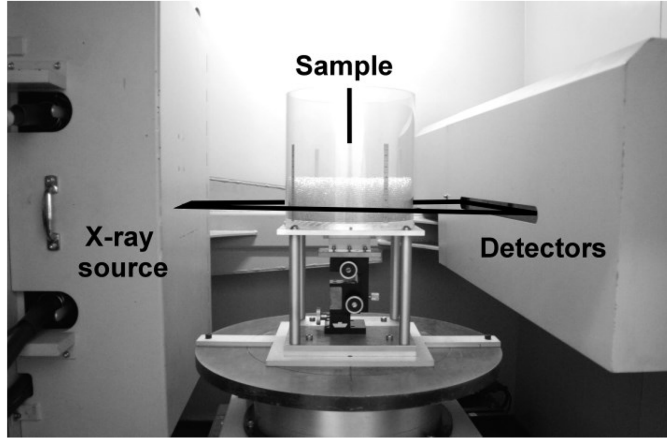

Figure 1. Picture of test device place in X-Ray CT scanner.

the granular material on the trapdoor. The steel support was vertically extendable so the trapdoor could be move downward with a screw. The displacement value of the trapdoor was controlled with a gauge. The different positions of the trapdoor corresponded to vertical displacement $\delta$ of, $0 \mathrm{~mm}, 0.1 \mathrm{~mm}, 1 \mathrm{~mm}$, $2 \mathrm{~mm}, 5 \mathrm{~mm}, 10 \mathrm{~mm}$ and $20 \mathrm{~mm}$.

\subsection{Granular material}

The granular material used in the tests was made of layers of single sized glass beads of, $80 \mathrm{~mm}$ height. Different samples were tested varying by the diameter of the beads: $2 \mathrm{~mm}, 4 \mathrm{~mm}$ and $6 \mathrm{~mm}$ respectively referred to as $\mathrm{G}_{2}, \mathrm{G}_{4}$ and $\mathrm{G}_{6}$. The density of the beads was of, $\gamma_{g}=2.52 \mathrm{~g} / \mathrm{cm}^{3}$. The range of void ratio was measured equal to, $e_{\min }=0.562$ and $e_{\max }=0.661$ and the sample were tested with an initial void ratio corresponding to the minimal value of, $e_{\min }$. The friction angles of glass beads were measured on a $\mathrm{G}_{2}$ sample of minimal void ratio, by dry triaxial compression test with a $25 \mathrm{kPa}$ confining pressure and found equal to, $\varphi_{p}=30^{\circ}$ for the peak value and $\varphi_{c}=24^{\circ}$ for the critical value. The friction angles of $\mathrm{G}_{4}$ and $\mathrm{G}_{6}$ samples were assumed to be the same as for $\mathrm{G}_{2}$.

\section{$2.3 \quad X$-Ray $C T$}

An industrial X-ray CT scanning system (Otani 2000, Otani 2002) of the Kumamoto University, Japan was used in this test series. It is a non-destructive method to investigate the spatial distribution of the densities. In this process, the sample is crossed by a collimated $\mathrm{x}$-ray beam in several directions by rotation and translation of the specimen. The intensities of the x-ray beams are measured and computed after the penetration of the specimen and an image of the section of the specimen can be reconstructed. The image obtained gives the distribution of CT-values which is related to the densities in the specimen by the absorption coefficient of the x-ray beam for the scanning point. The X-ray CT system used here was equipped with $300 \mathrm{kV}$ x-ray tubes (TOSCANER - $23200 \mathrm{~min}$ TOSHIBA Corp., Kumamoto University, Japan).
Table 1. Cases studied.

\begin{tabular}{llll}
\hline Case number & 1 & 2 & 3 \\
\hline Effect of beads size & $\mathrm{T}_{1} \times \mathrm{G}_{2}$ & $\mathrm{~T}_{1} \times \mathrm{G}_{4}$ & $\mathrm{~T}_{1} \times \mathrm{G}_{6}$ \\
Effect of trapdoor size & $\mathrm{T}_{1} \times \mathrm{G}_{4}$ & $\mathrm{~T}_{2} \times \mathrm{G}_{4}$ & $\mathrm{~T}_{3} \times \mathrm{G}_{4}$ \\
\hline
\end{tabular}

\subsection{Cases studied}

As presented before, 3 different trapdoors and 3 different granular samples were used in the tests, resulting in a total of 5 different test cases: a series of tests focusing on beads size using trapdoor $T_{1}$, a series of tests focusing on trapdoor shape using samples of $\mathrm{G}_{4}$. The different test cases are summarized in Table 1.

For each case, the measurement of the force and the CT scanning were conducted on separated tests. The tests for the force measurement were repeated 5 times. The test for CT scanning was conducted only once per case and for the trapdoor displacement $\delta$ of, $0 \mathrm{~mm}, 2 \mathrm{~mm}, 5 \mathrm{~mm}, 10 \mathrm{~mm}$.

\subsection{Method of analysis of CT results}

Trapdoor tests were performed on sample containing glass beads and air voids. The $\mathrm{CT}$ images give a spatial distribution of CT value which depends on the value of the density in each pixel. It is necessary to determine a threshold CT value in order to decide if one given pixel corresponds to a glass beads or to an air void. The threshold $\mathrm{CT}$ value was fixed based on the method explained by Chevalier \& Otani (2011). With the threshold value, raw CT images were converted into binary images, where particles could be clearly identified. Then, mechanisms could be analyzed based on the comparison between images taken for different positions of trapdoor. More details about the data treatment can be found in Chevalier \& Otani (2011).

\section{EFFECT OF BEADS SIZE}

\subsection{Effect on load transfer}

The stress on trapdoor was measured for several positions of the trapdoors. In each case, the initial mean stress on the trapdoor, resulting from the weight of the initial height of glass beads, was of, $1.5 \mathrm{kPa}$ approximately. The amplitude of the load transfer in the granular material was quantified by a stress ratio. The stress ratio is the ratio of the mean stress on trapdoor to the initial stress value.

The curve of stress ratio vs. displacement is shown on Fig. 2 for glass beads $\mathrm{G}_{2}, \mathrm{G}_{4}$ and $\mathrm{G}_{6}$. The response of the granular layer to the displacement of the trapdoor broke down into 2 successive phases. First, as soon as the trapdoor is moved downward, a sudden important decrease of the stress was measured, as observed in other trapdoor studies (Chevalier \& Otani 


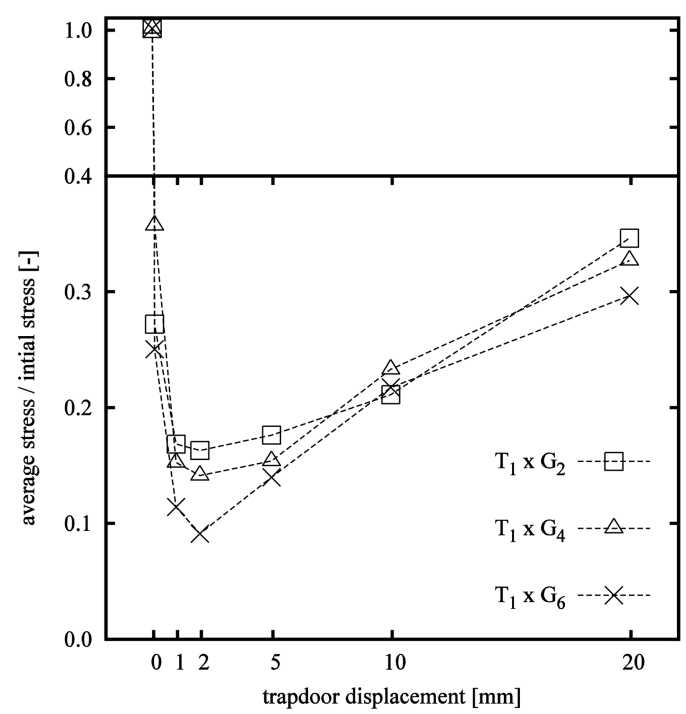

Figure 2. Stress ratio vs. trapdoor displacement for samples $\mathrm{G}_{2}, \mathrm{G}_{4}$ and $\mathrm{G}_{6}$ with trapdoor $\mathrm{T}_{1}$.

2011, Chevalier et al. 2012). The minimal value of the stress was obtained for a trapdoor displacement of, $2 \mathrm{~mm}$. Second, from $2 \mathrm{~mm}$ to $20 \mathrm{~mm}$ displacement, the mean stress on trapdoor increased slightly, still remaining in much smaller values than initial stress.

It could be noticed that, the influence of glass beads size on the minimal stress was clearly identified: the minimal stress increased as glass beads size decreased. Minimal stress ratios were equal to $8 \%$ for $\mathrm{G}_{6}, 14 \%$ for $G_{4}$ and $16 \%$ for $G_{2}$. Based on the results, it can be assumed that the minimal stress ratio converge to a limit as glass beads size is small enough. For the increase phase, the different sample could not be classed as easily as for minimal stress ratio. The stress ratio were ordered very differently for $10 \mathrm{~mm}$ and $20 \mathrm{~mm}$ displacement, so that no clear effect of glass beads size could be observed.

\subsection{Propagation of the disturbance}

The displacement of the trapdoor at the bottom of the granular layer creates a disturbance in the material, in terms of kinematics. For small displacement of the trapdoor, a small part of the material located in the vicinity of the trapdoor is affected and moves. Then, as the trapdoor displacement increases, the volume of disturbed material increases. At some point, a subsidence at the free surface of the layer can eventually be observed. Based on the binary images obtained from CT scanning, the volume disturbed by the trapdoor displacement could be measured.

For a better understanding, the measured volume was divided by the volume of material located above the trapdoor to obtain a disturbed volume ratio $V_{R}$ (Fig. 3). The Figure 4 shows the variation of the volume ratio vs trapdoor displacement for glass beads $\mathrm{G}_{2}$, $\mathrm{G}_{4}$ and $\mathrm{G}_{6}$.

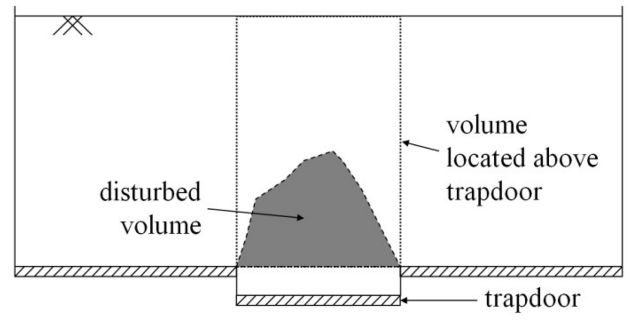

Figure 3. Definition of the volume ratio.

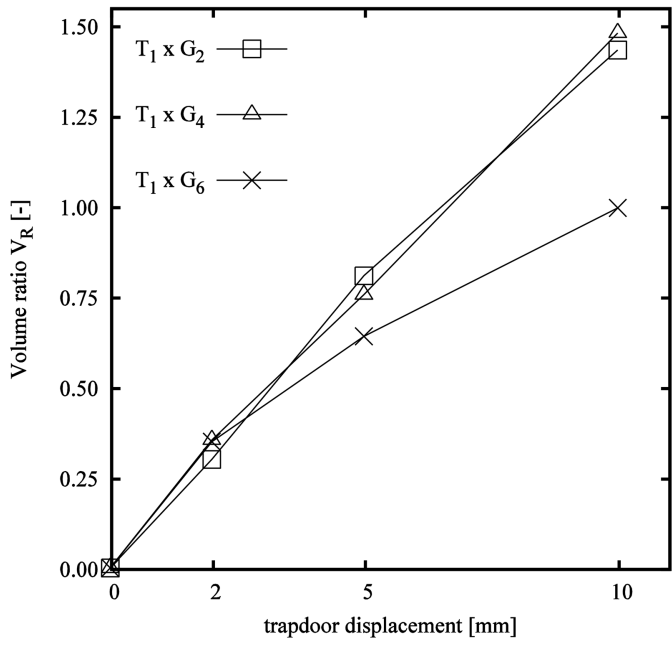

Figure 4. Volume ratio $V_{R}$ vs. trapdoor displacement for samples $\mathrm{G}_{2}, \mathrm{G}_{4}$ and $\mathrm{G}_{6}$ with trapdoor $\mathrm{T}_{1}$.

It was observed that the disturbed volume ratio increased as the trapdoor was moved. The disturbance due to the displacement of the trapdoor progressively propagated inside the granular layer. Until a trapdoor displacement of, $2 \mathrm{~mm}$, the disturbed volume ratio was very similar for the three sizes of glass beads. Then, for 5 and $10 \mathrm{~mm}$ displacement, a difference could be observed between $6 \mathrm{~mm}$ beads $\left(\mathrm{G}_{6}\right)$ on one side and 2 and $4 \mathrm{~mm}$ beads $\left(\mathrm{G}_{2}, \mathrm{G}_{4}\right)$ on the other side.

For $\mathrm{G}_{6}$, the disturbed volume ratio was smaller, suggesting that the propagation of the disturbance inside the granular material was more difficult. At the end of the test, $V_{R}$ is close to a value of, 1.0, which means that the volume affected by the movement of the trapdoor was equivalent to the volume above the trapdoor. For $\mathrm{G}_{2}$ and $\mathrm{G}_{4}$, the volume ratio at the end of test is close to 1.5: the disturbance due to trapdoor movement propagated in the same way with both size of the glass beads.

\subsection{Shape of the disturbance}

The shape of the horizontal cross section of the disturbed volume was measured with the circularity parameter $C$ defined by

$C=\frac{4 \pi A}{P^{2}}$ 

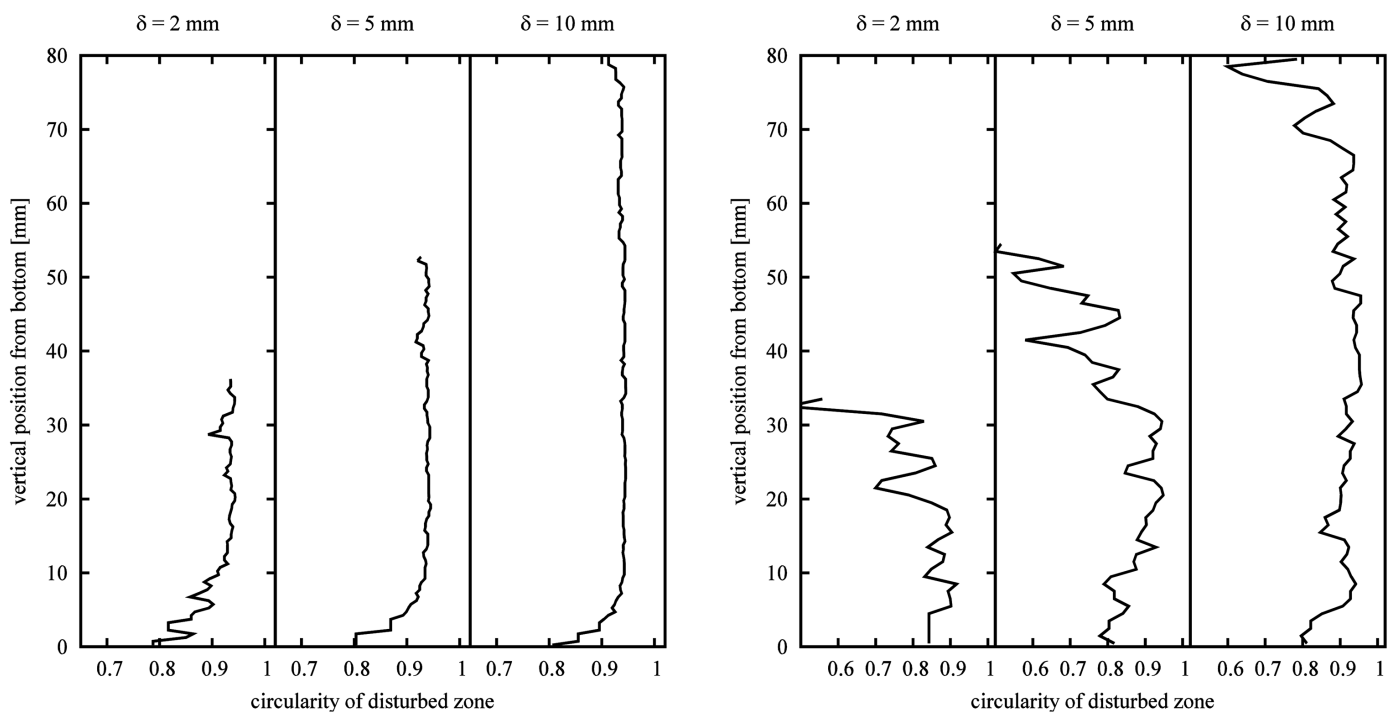

Figure 5. Circularity of disturbed volume in horizontal cross sections for $\mathrm{G}_{2}$ (left) and $\mathrm{G}_{6}$ (right).

where $A$ is the area of the cross section and $P$ its perimeter. $C=1$ for a circular cross section and $C=$ $\pi / 4$ for a square cross section (shape of trapdoor $T_{1}$ ). The circularity of the cross sections of disturbed volume vs. vertical position $z$ of the cross section are shown on Figure 5 for glass beads $\mathrm{G}_{2}$ and $\mathrm{G}_{6}$ for $\delta=2$, 5 and $10 \mathrm{~mm}$.

First, it can be noticed that the disturbed volume propagated from the bottom to the free surface of the layer as the trapdoor displacement increased. The propagation was similar for both size $G_{2}$ and $G_{6}$. Obviously, the circularity of disturbed volume was close to the value for square near the bottom of the test box.

For glass beads $\mathrm{G}_{2}$, as $z$ increased, $C$ tended to a plateau value of 0.95 , which is very close to the value for a circle. For glass beads $\mathrm{G}_{6}$, the variation of $C$ vs. $z$ is less smooth than for $\mathrm{G}_{2}$, and the plateau value of 0.95 is reached for $\delta=10 \mathrm{~mm}$ only.

The test results clearly showed that the failure planes bounding the disturbed volume of the granular material can make path among the grains in an easier way with finer grains. This observation has to be correlated with the influence of beads size on the load on the trapdoor, especially for small displacement of trapdoor.

\section{EFFECT OF TRAPDOOR SHAPE}

\subsection{Effect of load transfer}

The curves of the stress ratio vs. trapdoor displacement for the three cases of trapdoors are shown on Figure 6. A clear influence of trapdoor aspect ratio could be observed on the amplitude of the load transfer in granular layer.

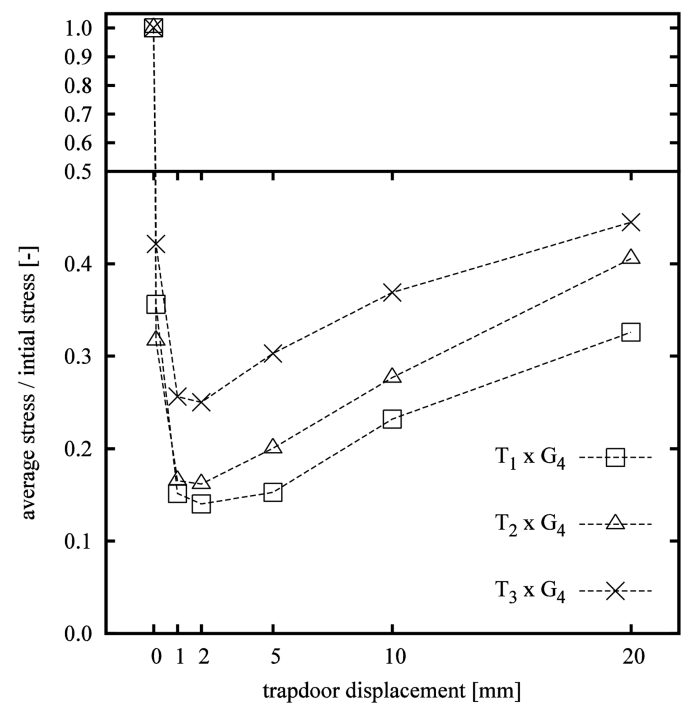

Figure 6. Stress ratio vs. trapdoor displacement for trapdoors $T_{1}, T_{2}$ and $T_{3}$ and with glass beads $G_{4}$.

The stress ratio increased with the aspect ratio of the trapdoor, for any displacement of the trapdoor: arching effect is more effective in terms of load transfer, if aspect ratio is small (close to the square in our case). It can be noticed that minimal stress ratios obtained with $\mathrm{T}_{1}(21 \%)$ and $\mathrm{T}_{2}(24 \%)$ were very close. As before, the two successive 'decreaseincrease' phases in the response of the granular layer could still be observed.

As the value of aspect ratio changes, the 3dimensional configuration (low aspect ratio) changes to a 2-dimensional configuration of the trapdoor 
problem (high aspect ratio). Because of the reduced number of values of aspect ratios used here, no clear transition between 3-dimensional and 2-dimensional conditions could be observed.

\subsection{Propagation of the disturbance}

From the CT images, binary images obtained for different positions of the trapdoor were compared and a volume ratio $V_{R}$ could be identified and measured (Fig. 3). $V_{R}$ is the ratio of the volume of disturbed zone to the volume of material located above trapdoor in the initial state. The curves showing the variations of $V_{R}$ vs. trapdoor displacement $\delta$ are given on Fig. 7.

The volume ratio obtained for the cases of trapdoors $T_{1}$ and $T_{2}$ were very close, suggesting a similar propagation of the disturbance in the granular layer. On the other side, the volume ratio obtained for $\mathrm{T}_{3}$ was much higher. This result may suggest a transition between 2-dimensional and 3-dimensional

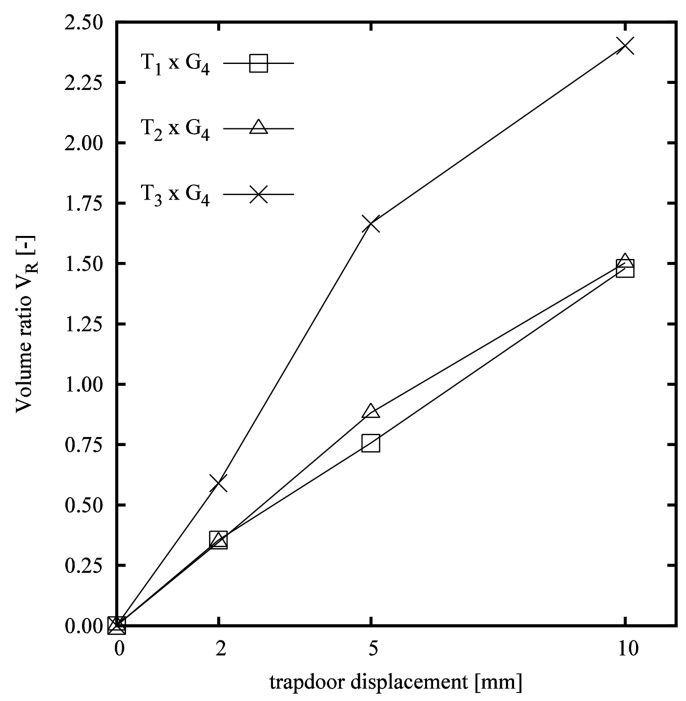

Figure 7. Volume ratio vs. trapdoor displacement for trapdoors $T_{1}, T_{2}$ and $T_{3}$ and with glass beads $G_{4}$. condition of trapdoor problem between $T_{1}$ and $T_{2}$ on one side and $\mathrm{T}_{3}$ on the other side. However, additional aspect ratios should be tested in order to confirm this assumption.

\subsection{Shape of the disturbance}

Horizontal cross sections of disturbed volume were analyzed with the circularity parameter (Eq. 1). The variation of the circularity $C$ from the bottom to the top of the granular layer is shown on Fig. 8 for trapdoor $\mathrm{T}_{1}$ and $\mathrm{T}_{3}$. The circularity of square shaped trapdoor $\mathrm{T}_{1}$ is equal to, $\pi / 4 \approx 0.78$ and equal to, $3 \pi / 16 \approx 0.59$ for $\mathrm{T}_{3}$.

For $\mathrm{T}_{1}$, a plateau value of circularity $C=0.95$, was reached for any value of trapdoor displacement $\delta$ and at a distance of, 5 to $10 \mathrm{~mm}$ from the bottom of the test box. On the contrary, for $\mathrm{T}_{3}$, the same plateau value of circularity was reached for $\delta \geq 5 \mathrm{~mm}$, and in the very upper part of the granular layer only. In both cases, the shape of horizontal cross section of disturbed zone changed from trapdoor shape (at the bottom of test box) to a circle shape at a distance large enough from the bottom of test box. As the aspect ratio increased, the distance necessary to observe a circle cross section increased, as well as the trapdoor displacement.

\section{CONCLUSION}

A parametric study of the trapdoor problem was conducted on particle size and trapdoor aspect ratio. The experimental tests included force measurement on the trapdoor and also CT scanning of the samples, for different positions of the trapdoor.

The results obtained showed that the amplitude of the load transfer resulting from arching effect depends on the size of the grains in the granular layer. When particles are fine enough compared to trapdoor size, this effect vanishes. During trapdoor test, the displacement of trapdoor induces a disturbance inside granular material. This disturbance propagates upward in the layer as trapdoor displacement increases.
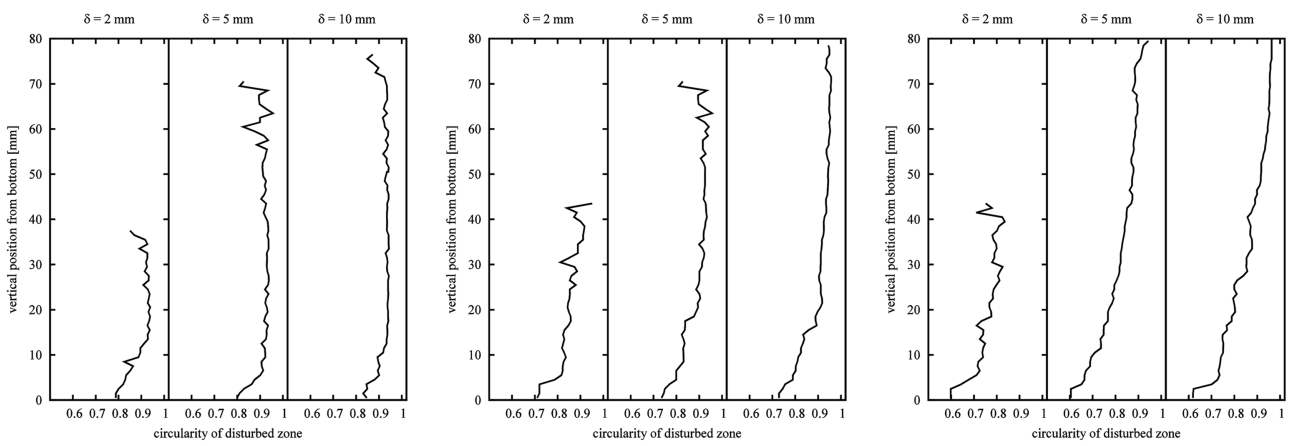

Figure 8. Circularity of disturbed volume in horizontal cross sections for $\mathrm{T}_{1}$ (left), $\mathrm{T}_{2}$ (middle) and $\mathrm{T}_{3}$ (right), with glass beads $\mathrm{G}_{4}$. 
As a result, a disturbed volume appears in the granular material. The shape of the disturbed volume is influenced by grain size, especially for coarse grains and for small displacement of trapdoor. With coarse grains, the failure planes are more difficult to initiate. When trapdoor displacement are large enough, or particles fine enough, the shape of horizontal cross sections of the disturbed volume becomes close to a circle.

Aspect ratio of trapdoor is another important parameter influencing arching effect. For a given aspect ratio, there is a transition in trapdoor problem between 3-dimensional conditions and 2-dimensional conditions. 3 values of aspect ratios were studied here varying from $1: 1$ to $3: 1$. The results didn't make this transition appear clearly, even if similarities could be observed between aspect ratio $1: 1$ and $2: 1$, contrary to $3: 1$

The amplitude of load transfer clearly decreases as aspect ratio increases. It was also observed that for trapdoor displacement large enough and at a distance from the trapdoor large enough, the shape of the disturbance propagating in the granular layer still tended to a circle shape.

The test results presented here involved a model granular material: samples of single sized glass beads. This material presented interesting properties for the observation in $\mathrm{x}$-ray $\mathrm{CT}$. It would be interesting to confirm the vanishing of size effect for finer particles. Real geomaterials with higher friction angle could be used. In order to quantify the transition between 3-dimensional and 2-dimensional conditions of the test, other intermediate aspect ratio could be tested.

\section{ACKNOWLEDGEMENT}

This research was conducted with a Grant-in-Aid of the Japanese Society for the Promotion of Science (JSPS 20.08818).

\section{REFERENCES}

Chevalier, B.; Combe, G. \& Villard, P. (2012), Experimental and discrete element modeling studies of the trapdoor problem: Influence of the macro-mechanical frictional parameters, Acta Geotechnica, 7(1):15-39.

Chevalier, B. \& Otani, J. (2011), Arching observation in threedimensional trapdoor problem with X-ray CT and discrete element method, Soils and Foundations, 51(3):459-469.

Dewoolkar, M. M.; Santichaianant, K. \& Ko, H.-Y. (2007), Centrifuge model of granular soil response over active circular trapdoors, Soils and Foundations, 45(5):931-945.

Pardo, G. S. \& Sáez, E. (2014), Experimental and numerical study of arching soil effect in coarse sand, Computers and Geotechnics, 57:75-84.

Tanaka, T. \& Sakai, T. (1993), Progressive failure and scale effect of trap-door problems with granular materials, Soils and Foundations, 33(1):11-22.

Terzaghi, K. (1936), Stress distribution in dry and saturated sand above a yielding trap-door, in Proceedings of International Conference of Soil Mechanics, Harvard University, Cambridge, pp. 307-311.

Vardoulakis, I.; Graf, B. \& Gudehus, G. (1981), Trap-door problem with dry sand: a statical approach based upon model test kinematics, International Journal for Numerical and Analytical Methods in Geomechanics, 5:57-78.

Vardoulakis, I.; Vairaktaris, E. \& Papamichos, E. (2004), Subsidence diffusion-convection: I. The direct problem, Computational Methods In Applied Mechanical Engineering, 193:2745-2760. 